\title{
Synergistic effect of rhein in combination with ampicillin or oxacillin against methicillin-resistant Staphylococcus aureus
}

\author{
DAE-KI JOUNG ${ }^{1}$, HEE JOUNG ${ }^{1}$, DA-WUN YANG ${ }^{1}$, DONG-YEUL KWON ${ }^{2}$, JANG-GI CHOI $^{2}$, \\ SEO WOO $^{3}$, DONG-YOUNG SHIN ${ }^{3}$, OH-HYUN KWEON ${ }^{4}$, KEE-TAE KWEON $^{5}$ and DONG-WON SHIN ${ }^{1}$ \\ ${ }^{1}$ Department of Oriental Medicine Resources, College of Bio Industry Science, \\ Sunchon National University, Sunchon, Jeonnam 540-742; ${ }^{2}$ Department of Oriental Pharmacy, \\ College of Pharmacy, Wonkwang Oriental Medicines Research Institute, Wonkwang University, Iksan, \\ Jeonbuk 570-749; ${ }^{3}$ Department of Development in Plant Resources, College of Bio Industry Science, \\ Sunchon National University, Sunchon, Jeonnam 540-742; ${ }^{4}$ Department of Physical Therapy, Andong Science College, \\ Seohu-Myeon, Andong-Si, Gyongbuk 760-820; ${ }^{5}$ Ministry of Health and Welfare Office for Healthcare \\ Policy Division of Traditional Korean Medicine, Jongro-gu, Seoul 110-793, Republic of Korea
}

Received October 22, 2011; Accepted December 28, 2011

DOI: $10.3892 /$ etm.2012.459

\begin{abstract}
Methicillin-resistant Staphylococcus aureus (MRSA) is a bacterium responsible for a number of infections in humans that are difficult to treat, and as a result, is a substantial contributor to morbidity and mortality. In the present study, in search of natural products capable of inhibiting this multidrug-resistant bacterium, we investigated the antimicrobial activity of rhein isolated from Rheum palmatum L. (Polygonaceae) against 16 different strains of the bacterium. New antimicrobial activity was found using the paper disc diffusion method [minimal inhibitory concentrations (MICs)], MTT test and checkerboard dilution test. Against the 16 strains, the disc diffusion test was in the range of 20-29 $\mathrm{mm}$ and the MICs of rhein were in the range of 7.8-31.25 $\mu \mathrm{g} / \mathrm{ml}$. From these results we performed the checkerboard test to determine the synergism of rhein in combination with ampicillin (AM) or oxacillin (OX) against all strains. The combined activity of rhein and the two antimicrobial agents (AM and OX) against all strains resulted in a fractional inhibitory concentration index ranging from $0.28-1$ and from $0.18-1$, respectively. The effect of rhein with AM and OX was found to be synergistic or partially synergistic. We found that rhein reduced the MICs of AM and OX. Rhein in combination with AM or OX could lead to the development of new combinations of antibiotics against MRSA infection.
\end{abstract}

Correspondence to: Dr Dong-Won Shin, Department of Oriental Medicine Resources, College of Bio Industry Science, Sunchon National University, Sunchon, Jeonnam 540-742, Republic of Korea E-mail:sdw@sunchon.ac.kr

Key words: antibacterial, methicillin-resistant Staphylococcus aureus, rhein, Rheum palmatum

\section{Introduction}

Methicillin-resistant Staphylococcus aureus (MRSA) is a serious and urgent clinical problem worldwide. Few new drugs are available against MRSA, since it has the ability to acquire resistance to most antibiotics. An effective method for treating MRSA may be to develop antibiotics from natural products which are likely to have lower toxicity and fewer side effects. Rheum palmatum, popularly known as Dahuang, has traditionally been used as an oriental folk medicine. Rhein naturally occurs in anthraquinone (1,3,8-trihydroxy-6-methyl anthraquinone) (Fig. 1), which is found in Rheum palmatum and related plants such as rhubarb. Anthraquinone components include aloe-emodin, rhein, emodin, chrysophanol and physcion (1). Rhein is used for treating numerous complaints, including constipation, jaundice, gastrointestinal hemorrhage and ulcers (2). It has also been shown to have good antitumor (3-6) and anti-inflammatory properties (7,8), anticancer (9), purgative (10), nephric protection $(11,12)$, liver protection (13), antimicrobial and hemostatic properties $(14,15)$. As such, it has been widely used for the treatment of gastrointestinal diseases, hepatitis and chronic renal failure. However, little is known about its antimicrobial effects on MRSA. Thus, we present the current study demonstrating the antimicrobial activity of rhein against MRSA and methicillin-sensitive S. aureus (MSSA) strains, as well as its synergistic effect.

\section{Materials and methods}

Test microorganisms. Among the 16 S. aureus strains used in this study, 14 clinical isolates of MRSA were obtained from 14 different patients at Wonkwang University Hospital (Iksan, Republic of Korea). The other two strains used were $S$. aureus ATCC 33591 (methicillin-resistant strain) and S. aureus ATCC 25923 (methicillin-susceptible strain). ATCC 25923 (American Type Culture Collection, Manassas, VA, USA) and ATCC 33591 were commercially purchased. Before use, all 


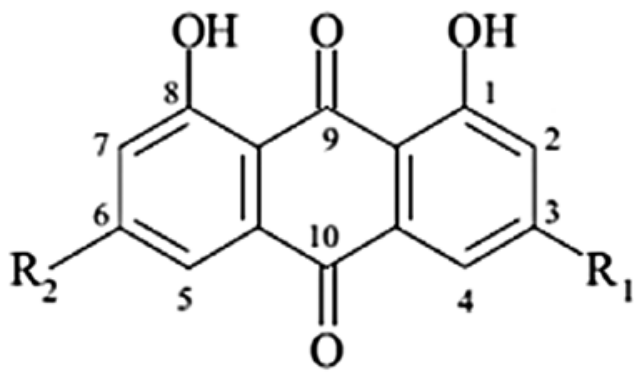

Figure 1. Structures of important anthraquinone derivatives. Rhein: $\mathrm{R}_{1}=\mathrm{COOH} ; \mathrm{R}_{2}=\mathrm{H}$. Aloe-emodin: $\mathrm{R}_{1}=\mathrm{CH}_{2} \mathrm{OH} ; \mathrm{R}_{2}=\mathrm{H}$. Emodin: $\mathrm{R}_{1}=\mathrm{CH}_{3}$; $\mathrm{R}_{2}=\mathrm{OH}$. Chrysophanol: $\mathrm{R}_{1}=\mathrm{CH}_{3} ; \mathrm{R}_{2}=\mathrm{H}$. Physcion: $\mathrm{R}_{1}=\mathrm{CH}_{3} ; \mathrm{R}_{2}=\mathrm{OCH}_{3}$

bacteria were stored in $30 \%$ glycerol and frozen at $-70^{\circ} \mathrm{C}$. The bacteria were cultured in Mueller-Hinton broth (MHB) and Mueller-Hinton agar (MHA) (Difco Laboratories, Baltimore, MD, USA). Bacteria were suspended in MHB and then incubated at $37^{\circ} \mathrm{C}$ for $24 \mathrm{~h}$.

Disc diffusion method. The disc diffusion method was performed as described by the Clinical and Laboratory Standards Institute (CLSI) and by using a modified agar well diffusion method (CLSI, 2001) (16). Bacterial strains grown on $\mathrm{MHA}$ at $37^{\circ} \mathrm{C}$ for $18 \mathrm{~h}$ were suspended in MHB and adjusted to a turbidity of 0.5 on the McFarland standard scale (approximately $1.5 \times 10^{8} \mathrm{CFU} / \mathrm{ml}$ ). The MHA was poured into Petri dishes and inoculated with $100 \mu 1$ of the suspension. Sterile paper discs (diameter, 6 mm; Tokyo Roshi Kaihsa, Japan) were punched into the agar and each well was filled with 500 and $250 \mu \mathrm{g}$ of each drug. The dissolution of the ampicillin (AM), oxacillin (OX) and rhein was facilitated by the addition of $50 \%$ (v/v) DMSO (50\% DMSO was not active against all strains) (DMSO; Sigma, St. Louis, MO, USA). AM and OX were used as the positive controls, and the discs treated with DMSO were used as the negative control. The plates were placed in an incubator at $37^{\circ} \mathrm{C}$ for $18 \mathrm{~h}$ in the dark. The inhibition zone diameter around each of the discs was measured and recorded at the end of the incubation period.

Minimum inhibitory concentration. The minimum inhibitory concentration (MIC) was determined using the broth microdilution method according to the CLSI guidelines, 2000 (17). Briefly, the inoculation of the microorganisms was carried out on $24 \mathrm{~h}$ broth cultures, and the suspensions were adjusted to a $0.5 \mathrm{McF}$ arland standard turbidity (approximately $1.5 \times 10^{8} \mathrm{CFU} /$ $\mathrm{ml}$ ). Final inoculum size was adjusted to $1.5 \times 10^{6} \mathrm{CFU} / \mathrm{ml}$. These serially diluted cultures were then incubated at $37^{\circ} \mathrm{C}$ for $18 \mathrm{~h}$. MIC was defined at the lowest concentration of AM, OX and rhein. At the end of the incubation period, the well plates were visually examined for turbidity. Cloudiness indicated that bacterial growth had not been inhibited by the concentration of the antimicrobial agent contained in the medium.

Checkerboard dilution test. The synergistic combinations were investigated in the preliminary checkerboard method performed using the MRSA and MSSA strains, and one clinical isolate strain via MIC determination, according to
Table I. The S. aureus strains used in the experiments.

\begin{tabular}{llcc}
\hline $\begin{array}{l}\text { S. aureus } \\
\text { strain }\end{array}$ & Class & mecA gene & $\begin{array}{c}\text { Antibiotic } \\
\text { resistance pattern }\end{array}$ \\
\hline ATCC 25923 & MSSA & - & - \\
ATCC 33591 & MRSA & + & AM, OX \\
DPS-1 & MRSA & + & AM, OX \\
DPS-2 & MRSA & + & AM, OX \\
DPS-3 & MRSA & + & AM, OX \\
DPS-4 & MRSA & + & AM, OX \\
DPS-5 & MRSA & + & AM, OX \\
DPS-6 & MRSA & + & AM, OX \\
DPS-7 & MRSA & + & AM, OX \\
DPS-8 & MRSA & + & AM, OX \\
DPS-9 & MRSA & + & AM, OX \\
DPS-10 & MRSA & + & AM, OX \\
DPS-11 & MRSA & + & AM, OX \\
DPS-12 & MRSA & + & AM, OX \\
DPS-13 & MRSA & + & AM, OX \\
DPS-14 & MRSA & + & AM, OX \\
\hline
\end{tabular}

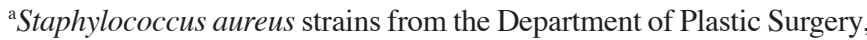
Wonkwang University Hospital, Iksan, Korea. (+), positive; (-), negative; AM, ampicillin; OX, oxacillin.

the CLSI guidelines (18). The MIC was defined as the lowest concentration of drug alone or in combination that inhibited the visible growth. The in vitro interaction was quantified by determining the fractional inhibitory concentration (FIC). The FIC index was calculated as follows: $\mathrm{FIC}=(\mathrm{MIC}$ of drug $\mathrm{A}$ in combination/MIC of drug $\mathrm{A}$ alone $)+(\mathrm{MIC}$ of drug $\mathrm{B}$ in combination/MIC of drug B alone). FIC indices (FICIs) were interpreted as follows: $<0.5$, synergy; 0.5-0.75, partial synergy; 0.76-1.0, additive effect; >1.0-4.0, indifference; and >4.0, antagonism. Finally, the varying rates of synergy between the two agents were determined. All experiments were independently repeated three times.

Colorimetric assay using MTT test. A colorimetric assay based on MTT for the rapid detection of the presence of bacteria was performed as previously described (19-21). Briefly, a stock solution of $5 \mathrm{mg} / \mathrm{ml}$ MTT (Sigma) was prepared in phosphatebuffered saline and kept at $-70^{\circ} \mathrm{C}$. A final concentration of $1 \mathrm{mg} / \mathrm{ml}$ of MTT was used in the assay. Following $24 \mathrm{~h}$ of incubation a $37^{\circ} \mathrm{C}, 20 \mu \mathrm{l}$ of the yellow MTT was added to the 96-well microtiter plate ( $0.3 \mathrm{ml}$ volume) and incubated for an additional $20 \mathrm{~min}$. The presence of a blue color indicated the presence of bacteria.

\section{Results}

Table I shows the $S$. aureus strains used in the experiments. Table II shows the means of inhibition zones produced against the tested bacteria ranged between 20 and $29 \mathrm{~mm}$. The growth of all the tested strains of MRSA and MSSA was inhibited at 500 and $250 \mu \mathrm{g} /$ disc. As shown in Table III, rhein demon- 
Table II. The antimicrobial activity (as inhibitory zone diameters) of rhein, AM and OX against the S. aureus strain.

\begin{tabular}{|c|c|c|c|c|c|c|}
\hline \multirow[b]{3}{*}{ S. aureus strain } & \multicolumn{6}{|c|}{ Zone of inhibition (mm) } \\
\hline & \multicolumn{2}{|c|}{ Rhein $(\mu \mathrm{g} / \mathrm{ml})$} & \multicolumn{2}{|c|}{ Ampicillin $(\mu \mathrm{g} / \mathrm{ml})$} & \multicolumn{2}{|c|}{ Oxacillin $(\mu \mathrm{g} / \mathrm{ml})$} \\
\hline & 500 & 250 & 500 & 250 & 500 & 250 \\
\hline ATCC 25923 & 25 & 22 & 43 & 41 & 37 & 35 \\
\hline ATCC 33591 & 29 & 23 & 16 & 15 & 18 & 15 \\
\hline DPS- $1^{\text {a }}$ & 19 & 14 & 18 & 14 & ND & ND \\
\hline DPS-2 & 25 & 20 & 18 & 17 & 17 & 15 \\
\hline DPS-3 & 21 & 17 & 21 & 16 & ND & ND \\
\hline DPS-4 & 21 & 18 & 20 & 15 & ND & ND \\
\hline DPS-5 & 22 & 18 & 17 & 14 & ND & ND \\
\hline DPS-6 & 20 & 16 & 17 & 14 & ND & ND \\
\hline DPS-7 & 21 & 18 & 11 & 10 & ND & ND \\
\hline DPS-8 & 21 & 17 & 12 & 11 & ND & ND \\
\hline DPS-9 & 23 & 19 & 13 & 12 & ND & ND \\
\hline DPS-10 & 25 & 20 & 12 & 11 & ND & ND \\
\hline DPS-11 & 25 & 21 & 11 & 9 & ND & ND \\
\hline DPS-12 & 24 & 20 & 12 & 10 & ND & ND \\
\hline DPS-13 & 24 & 19 & 18 & 14 & ND & ND \\
\hline DPS-14 & 20 & 16 & 15 & 11 & ND & ND \\
\hline
\end{tabular}

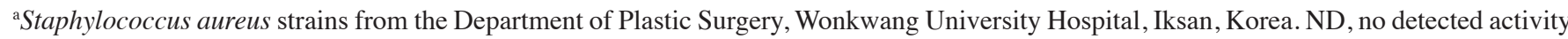
at this concentration. AM, ampicillin; OX, oxacillin.

Table III. The MICs of rhein, AM and OX against the S. aureus strain.

\begin{tabular}{lccc}
\hline & \multicolumn{3}{c}{ MICs $(\mu \mathrm{g} / \mathrm{ml})$} \\
\cline { 2 - 4 } S. aureus strain & Rhein & Ampicillin & Oxacillin \\
\hline ATCC25923 & 15.62 & 7.8 & 7.8 \\
ATCC33591 & 15.62 & 1,000 & 250 \\
DPS-1 & 15.62 & 31.25 & 500 \\
DPS-2 & 15.62 & 1,000 & 500 \\
DPS-3 & 15.62 & 31.25 & 500 \\
DPS-4 & 31.25 & 31.25 & 500 \\
DPS-5 & 7.8 & 31.25 & 500 \\
DPS-6 & 7.8 & 31.25 & 250 \\
DPS-7 & 31.25 & 250 & 500 \\
DPS-8 & 31.25 & 250 & 500 \\
DPS-9 & 31.25 & 125 & 500 \\
DPS-10 & 31.25 & 250 & 500 \\
DPS-11 & 31.25 & 250 & 500 \\
DPS-12 & 31.25 & 250 & 500 \\
DPS-13 & 7.8 & 31.25 & 1,000 \\
DPS-14 & 7.8 & 250 & 500 \\
\hline
\end{tabular}

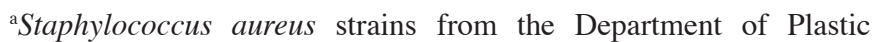
Surgery, Wonkwang University Hospital, Iksan, Korea. MIC, minimal inhibitory concentration; AM, ampicillin; OX, oxacillin.

strated antimicrobial activity against all the tested strains of MRSA as well as the MSSA strain. The MICs of rhein against S. aureus ranged from 7.8-31.25 $\mu \mathrm{g} / \mathrm{ml}$. As shown in Tables IV and $\mathrm{V}$, tests were performed to determine the action of rhein alone as well as its synergistic action with AM or OX against the MRSA clinical isolate, the standard MRSA strain or the standard MSSA strain. Rhein lowered the MICs of AM and OX against the MRSA strains. For the standard MSSA strain, rhein lowered the MICs of both AM and OX. The combined activity of rhein and the two antimicrobial agents (AM and OX) against all strains resulted in a FICI ranging from $0.28-1$ and from $0.18-1$, respectively. In conclusion, the combination effect of rhein with AM and OX was found to be synergistic or partially synergistic.

\section{Discussion}

In the present study, we investigated the antimicrobial activity of rhein against clinical isolates of MRSA and a standard MSSA strain. We report that the rhein agent also shows synergistic activity with AM and OX against MRSA. When combined, these antibiotic effects were dramatically increased. While the results obtained in the present study cannot currently be applied in clinical practice, we consider that the combination treatment of rhein isolated with AM or OX will prove to be helpful in treating MRSA and MSSA. Further medicinal, clinical and mechanism studies are required to verify the mechanisms by which rhein enhances the antibacterial activity. At present, the continued emergence of multi-drug resistant bacteria and the infectious diseases caused by them are serious global problems. It is hoped that it will be possible to reduce the use of existing antibacterial drugs and increase the use of natural product drugs, such as rhein. At this point in time, the product is still under investigation. In our study, rhein mark- 
Table IV. Result of the combined effect of rhein and AM against S. aureus.

\begin{tabular}{lccccc}
\hline & \multicolumn{3}{c}{ MICs $(\mu \mathrm{g} / \mathrm{ml})$} \\
\cline { 2 - 5 } S. aureus strain & Rhein alone & With AM & AM alone & With rhein & FICI \\
\hline ATCC 25923 & 15.62 & 3.9 & 7.8 & 1.95 & 0.5 \\
ATCC 33591 & 15.62 & 0.97 & 1,000 & 250 & 0.31 \\
DPS-1 & 15.62 & 3.5 & 31.25 & 7.8 & 0.5 \\
DPS-2 & 15.62 & 3.9 & 1,000 & 250 & 0.5 \\
DPS-3 & 15.62 & 7.8 & 31.25 & 0.97 & 0.53 \\
DPS-4 & 31.25 & 15.62 & 31.25 & 0.97 & 0.53 \\
DPS-5 & 7.8 & 3.9 & 31.25 & 15.62 & 1 \\
DPS-6 & 7.8 & 3.9 & 31.25 & 7.9 & 0.75 \\
DPS-7 & 31.25 & 7.8 & 250 & 62.5 & 0.5 \\
DPS-8 & 31.25 & 7.8 & 250 & 7.8 & 0.28 \\
DPS-9 & 31.25 & 7.8 & 125 & 3.9 & 0.28 \\
DPS-10 & 31.25 & 7.8 & 250 & 15.62 & 0.28 \\
DPS-11 & 31.25 & 7.8 & 250 & 62.5 & 0.31 \\
DPS-12 & 31.25 & 3.9 & 250 & 15.62 & 0.5 \\
DPS-13 & 7.8 & 0.48 & 250 & 125 & 1 \\
DPS-14 & 7.8 & & & & 0.56 \\
\hline
\end{tabular}

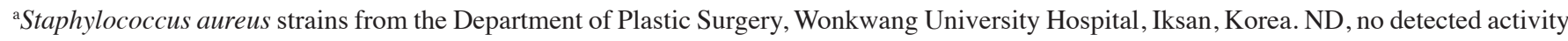
at this concentration; MIC, minimal inhibitory concentration; AM, ampicillin; FICI, fractional inhibitory concentration index.

Table V. Result of the combined effect of rhein and OX against S. aureus.

\begin{tabular}{|c|c|c|c|c|c|}
\hline \multirow[b]{2}{*}{ S. aureus strain } & \multicolumn{5}{|c|}{$\operatorname{MICs}(\mu \mathrm{g} / \mathrm{ml})$} \\
\hline & Rhein alone & With OX & OX alone & With rhein & FICI \\
\hline ATCC 25923 & 15.62 & 1.95 & 7.8 & 1.95 & 0.37 \\
\hline ATCC 33591 & 15.62 & 1.95 & 250 & 15.62 & 0.18 \\
\hline DPS- $1^{\text {a }}$ & 15.62 & 7.8 & 500 & 31.25 & 0.53 \\
\hline DPS-2 & 15.62 & 3.9 & 500 & 125 & 0.5 \\
\hline DPS-3 & 15.62 & 7.8 & 500 & 15.62 & 0.53 \\
\hline DPS-4 & 31.25 & 7.8 & 500 & 125 & 0.5 \\
\hline DPS-5 & 7.8 & 1.95 & 500 & 125 & 0.5 \\
\hline DPS-6 & 7.8 & 1.95 & 250 & 7.8 & 0.53 \\
\hline DPS-7 & 31.25 & 7.8 & 500 & 125 & 0.5 \\
\hline DPS-8 & 31.25 & 7.8 & 500 & 62.5 & 0.37 \\
\hline DPS-9 & 31.25 & 7.8 & 500 & 125 & 0.5 \\
\hline DPS-10 & 31.25 & 7.8 & 500 & 62.5 & 0.37 \\
\hline DPS-11 & 31.25 & 7.8 & 500 & 125 & 0.5 \\
\hline DPS-12 & 31.25 & 7.8 & 500 & 250 & 0.75 \\
\hline DPS-13 & 7.9 & 0.48 & 1,000 & 500 & 0.56 \\
\hline DPS-14 & 7.9 & 3.9 & 500 & 250 & 1 \\
\hline
\end{tabular}

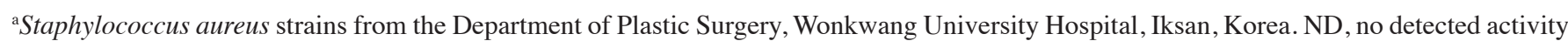
at this concentration; MIC, minimal inhibitory concentration; OX, oxacillin; FICI, fractional inhibitory concentration index.

edly lowered the MICs of AM and OX against the two MRSA strains and one MSSA strain. While the product is still under investigation, the present results are promising and may help to promote the use of natural products rather than antibiotics.

\section{Acknowledgements}

This study was supported by the Sunchon National University Research Fund (2011). 


\section{References}

1. Yan D, Ma Y, Shi R, Xu D and Zhang N: Pharmacokinetics of anthraquinones in Xiexin decoction and in different combinations of its constituent herbs. Phytother Res 23: 317-323, 2009.

2. Chinese Pharmacopoeia Commission: Pharmacopoeia of the People's Republic of China. Chemical Industry Press, Beijing, p17, 2005.

3. Dorsey JF and Kao GD: Aloe(-emodin) for cancer? More than just a comforting salve. Cancer Biol Ther 6: 89-90, 2007.

4. Huang Q, Lu G, Shen HM, Chung MC and Ong CN: Anti-cancer properties of anthraquinones from rhubarb. Med Res Rev 27: 609-630, 2007.

5. Lee HZ, Hsu SL, Liu MC and Wu CH: Effects and mechanisms of aloe-emodin on cell death in human lung squamous cell carcinoma. Eur J Pharmacol 431: 287-295, 2001.

6. Shi YQ, Fukai T, Sakagami H, Kuroda J, Miyaoka R, Tamura M, Yoshida N and Nomura T: Cytotoxic and DNA damage-inducing activities of low molecular weight phenols from rhubarb. Anticancer Res 21: 2847-2853, 2001.

7. Cuellar MJ, Giner RM, Recio MC, Manez S and Rios JL: Topical anti-inflammatory activity of some Asian medicinal plants used in dermatological disorders. Fitoterapia 72: 221-229, 2001.

8. Ding Y, Zhao L, Mei H, Zhang SL, Huang ZH, Duan YY and Ye P: Exploration of emodin to treat alpha-naphthylisothiocyanate-induced cholestatic hepatitis via anti-inflammatory pathway. Eur J Pharmacol 590: 377-386, 2008.

9. Cai J, Razzak A, Hering J, Saed A, Babcock TA, Helton S and Espat NJ: Feasibility evaluation of emodin (rhubarb extract) as an inhibitor of pancreatic cancer cell proliferation in vitro. JPEN J Parenter Enteral Nutr 32: 190-196, 2008.

10. Huang KC: The Pharmacology of Chinese Herbs. CRC Press, Boca Raton, FL, pp233-234, 1993.

11. Yokozawa T, Suzuki N, Okuda I, Oura H and Nishioka I: Changes in the urinary constituents in rats with chronic renal failure during oral administration of rhubarb extract. Chem Pharm Bull (Tokyo) 33: 4508-4514, 1985.
12. Wang J, Zhao Y, Xiao X, Li H, Zhao H, Zhang P and Jin C: Assessment of the renal protection and hepatotoxicity of rhubarb extract in rats. J Ethnopharmacol 124: 18-25, 2009.

13. Zhao YL, Wang JB, Zhou GD, Shan LM and Xiao XH: Investigations of free anthraquinones from rhubarb against $\alpha$-naphthylisothiocyanate-induced cholestatic liver injury in rats. Basic Clin Pharmacol Toxicol 104: 463-469, 2009.

14. Dictionary of Traditional Chinese Medicine: New Medical College of Jiangsu. 1st edition. People's Publishing Co. of Shanghai, p102, 1997.

15. WHO: Monographs on Selected Medicinal Plants. World Health Organization, Geneva, pp231-240, 1999.

16. Clinical and Laboratory Standards Institute: Performance standards for antimicrobial disk susceptibility tests. Approved standards. CLSI document M2 A7. Wayne, PA, 2001.

17. Clinical and Laboratory Standards Institute: Methods for dilution antimicrobial susceptibility tests for bacteria that grow aerobically. Approved standards. CLSI document M7-A5. Wayne, PA, 2000.

18. Mazumdor K, Dutta NK, Kumar KA and Dastidar SG: In vitro and in vivo synergism between tetracycline and the cardiovascular agent oxyfedrine HCI against common bacterial strains. Biol Pharm Bull 28: 713-717, 2005.

19. Abate G, Mshana RN and Miorner H: Evaluation of a colorimetric assay based on 3-(4,5-dimethylthiazol-2-yl)-2,5-diphenyl tetrazolium bromide (MTT) for rapid detection of rifampicin resistance in Mycobacterium tuberculosis. Int J Tuberc Lung Dis 2: 1011-1016, 1988.

20. Scheuber PH, Scheuber PH, Mossmann H, Beck G and Hammer DK: Direct skin test in highly sensitized guinea pigs for rapid and sensitive determination of staphylococcal enterotoxin B. Appl Environ Microbiol 46: 1351-1356, 1983.

21. Shi YJ, Chen J and Xu M: A new method for antimicrobial susceptibility testing of in vitro-cultured bacteria by means of resonance light scattering technique. J Microbiol Biotechnol 18: 118-123, 2008. 